\title{
Entrainement au contrôle inhibiteur et apprentissage en chimie dans le secondaire supérieur : Favoriser un changement de prévalence conceptuelle
}

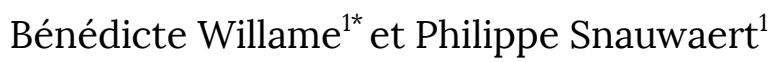

\begin{abstract}
RÉSUMÉ
L'apprentissage des modèles chimiques n'est pas simple d'autant plus que le passage du niveau macroscopique au niveau microscopique de la matière est compliqué par l'utilisation de représentations symboliques. Une habitude intuitive telle que « More A then more B » souvent utilisée chez les plus jeunes apprenants peut être abandonnée par les plus experts en chimie au profit de l'utilisation, parfois erronée, de modèles chimiques proposés lors du cursus scolaire. Dans le contexte de la dissolution d'un soluté dans un solvant, cette habitude est pourtant la stratégie de réponse efficace pour acquérir que le volume de solution est supérieur au volume de solvant et pouvoir ainsi calculer correctement la concentration en soluté (grandeur qui se rapporte au volume de solution). Notre recherche vise à améliorer l'apprentissage de ce concept, grandeur fondamentale en chimie. Nous avons élaboré un dispositif didactique expérimental basé sur l'utilisation d'outils permettant un conflit cognitif et un entrainement à la fonction exécutive de contrôle inhibiteur des conceptions non pertinentes dans le contexte de la dissolution d'un sel dans l'eau. L'objectif est de permettre une (ré)activation de la stratégie de réponse « More A then more $B$ » afin de la rendre prévalente dans ce contexte. Cette étude confronte les scores obtenus par deux groupes d'élèves de grade 10 (15-17 ans) : l'un soumis à une activité de remédiation impliquant un conflit perceptocognitif seul et un autre groupe ayant réalisé cette même activité, mais suivie d'un entrainement à l'inhibition des conceptions non pertinentes dans le contexte de la dissolution d'un sel dans l'eau et du calcul d'une concentration. Les résultats obtenus montrent un impact positif du conflit cognitif suivi d'un apprentissage métacognitif à l'inhibition pour l'acquisition d'un concept de chimie pour des élèves du secondaire. Ils mettent également en évidence que des liens cognitifs privilégiés semblent exister lors du changement de prévalence conceptuelle.
\end{abstract}

\footnotetext{
${ }^{1}$ Université de Namur, Unité de didactique de la chimie, Namur, Belgique

* Correspondance avec l'auteure : benedicte.willame@ismcouvin.eu
}

Pour citer cet article : Willame, B. et Snauwaert, P. (2018). Entrainement au contrôle inhibiteur et apprentissage en chimie dans le secondaire supérieur : Favoriser un changement de prévalence conceptuelle. Neuroéducation, 5(2), 73-92.

DOI: https://doi.org/10.24046/neuroed.20180502.73

Reçu le 31 octobre 2017. Révision reçue le 29 avril 2018.

Accepté le 10 mai 2018. Publié en ligne le 19 septembre 2018.

Neuroéducation, 5(2), 73-92

ISSN: 1929-1833

Tous droits réservés (C) 2018 - Association pour la recherche en neuroéducation 


\section{Introduction}

La chimie est une science qui a pour objectif de comprendre et d'expliquer la matière, ses comportements et ses modifications. Acquérir les modèles conçus par les chimistes n'est pas simple d'autant plus que le passage du niveau macroscopique au niveau microscopique en utilisant les représentations iconographiques complique leur apprentissage (Johnstone, 1993; Tsaparlis et Sevian, 2013).

Dans son laboratoire, le chimiste manipule des solutions qu'il prépare en dissolvant un soluté dans un solvant. La grandeur qui lui permet de connaitre la quantité de soluté qu'il a dissout par rapport au volume total de solution est fondamentale. Cette grandeur, appelée concentration chimique, impose au chimiste qui manipule la solution d'être conscient que le volume de solution est supérieur au volume de solvant. Dans un précédent article, nous avons proposé les résultats d'une étude diagnostique qui met en évidence que l'assimilation entre le volume de solvant et celui de solution est récurrente chez les apprenants (Willame et Snauwaert, 2015).

Notre recherche a pour objectif d'élaborer une stratégie didactique permettant de remédier aux difficultés d'apprentissage du concept de concentration chimique. Nous nous situons après l'apprentissage de ce concept. Les outils didactiques tels que ceux envisagés par les courants théoriques du changement conceptuel proposent de créer un conflit cognitif chez l'apprenant en difficulté afin que sa conception erronée soit remplacée par une conception plus conforme au savoir savant (Astolfi et al., 2008).

Limagerie fonctionnelle du cerveau a permis aux neuroscientifiques de mettre en évidence que réaliser un changement de conception consisterait davantage à inhiber les réseaux neuronaux menant à des réponses inappropriées (Houdé, 2004, 2016b; Masson, 2012, 2016). Le modèle récent de prévalence conceptuelle postule que plusieurs conceptions coexisteraient et qu'une inhibition des conceptions non pertinentes dans le contexte de la question permettrait de répondre correctement à la question (Potvin, 2013; Potvin, Sauriol et Riopel, 2015).

L'étude proposée dans cet article confronte les réponses apportées par des élèves à des questions sur la concentration chimique selon qu'ils aient ou non suivi des activités basées sur un conflit cognitif seul ou sur un conflit cognitif suivi d'un entrainement à la fonction exécutive de contrôle inhibiteur des conceptions non pertinentes.

Dans la première partie, nous aborderons les cadres théoriques de notre recherche : tant ceux liés aux modèles utilisés en chimie pour décrire la dissolution d'un soluté dans un solvant que les fondements des outils didactiques et neuroscientifiques qui nous ont permis d'élaborer les activités de remédiation. Dans la seconde partie, après avoir posé les questions de recherche, nous présentons la méthodologie adoptée pour mesurer limpact de nos outils. Les résultats et une discussion ainsi que des propositions pour l'enseignement feront l'objet de la troisième et dernière partie.

\section{Cadres théoriques de la recherche}

2.1 Les modèles chimiques utilisés pour expliquer la dissolution d'un soluté et calculer sa concentration au sein d'une solution

Peu avant d'étudier le concept de concentration, l'élève appréhende d'abord le phénomène de dissolution où un soluté est dissout dans un solvant pour former une solution. Cette dissolution visible au niveau macroscopique s'explique au niveau microscopique par des modèles proposés par les chimistes.

Pour expliquer la dissolution d'un composé ionique comme du sel de cuisine dans l'eau, le chimiste assimile le sel de cuisine à une substance pure, le chlorure de sodium. Les cristaux cubiques observables à l'échelle macroscopique sont décrits à l'échelle microscopique par un réseau d'ions chlorure $\mathrm{Cl}^{-}$et d'ions sodium $\mathrm{Na}^{+}$souvent représenté par l'alternance de boules vertes négatives et de boules noires positives (figure 1a).

La dissolution du chlorure de sodium dans l'eau peut être modélisée par une dissociation des ions du cristal. Les molécules d'eau s'orientent de telle façon que leur pôle négatif interagit avec les ions $\mathrm{Na}^{+}$et leur pôle positif avec les ions $\mathrm{Cl}^{-}$ (figure 1b). L'attraction entre les ions en surface du cristal et les dipôles des molécules d'eau étant supérieure à la force d'attraction électrostatique entre les ions de sodium et de chlorure, les ions $\mathrm{Na}^{+}$et $\mathrm{Cl}^{-}$passent en solution entourés d'une «sphère de solvatation» (figures 1c et 1d) (Arnaud, 1989; Nivaldo, 2011). La place occupée par les ions du sel dissout a fait augmenter le volume initial de liquide. Le volume de solution est supérieur au volume de solvant.

Pour expliquer la dissolution de composés covalents polaires tels que le saccharose (sucre ajouté dans le café) ou l'éthanol (alcool du vin), le modèle établi par les chimistes se réfère à des liaisons intermoléculaires comme les ponts hydrogène entre les pôles du solvant et ceux du soluté.

Pour aborder les niveaux macroscopique et microscopique (ou sous-microscopique), la représentation symbolique est très souvent utilisée en chimie. Les didacticiens de la chimie intègrent totalement cette représentation comme faisant partie des niveaux de savoir en chimie (Johnstone, 1993). Les choix réalisés dans les représentations de la matière sont variés. C'est ainsi que les représentations iconographiques telles que des formes géométriques sont utilisées pour représenter les solutions. Afin de représenter un soluté qui se dissout dans un solvant, la matière est souvent symbolisée par des ronds en deux dimensions (2D) qui, eux-mêmes, représentent des boules en 3D.

Les concentrations massiques et molaires sont celles principalement développées en grades 9 et 10 du secondaire supérieur en Belgique francophone. Celles-ci se définissent comme le rapport entre la masse (concentration massique) ou la quantité de matière (concentration molaire) et le volume de solution. En d'autres termes, la concentration chimique indique une proportion de soluté dans la solution. Il s'agit d'une grandeur intensive.

\footnotetext{
${ }^{1}$ Une grandeur intensive n'est pas dépendante de la taille, de l'étendue du système (Legrand, 2006). En tout point d'un système homogène, la mesure d'une grandeur intensive est identique.
} 


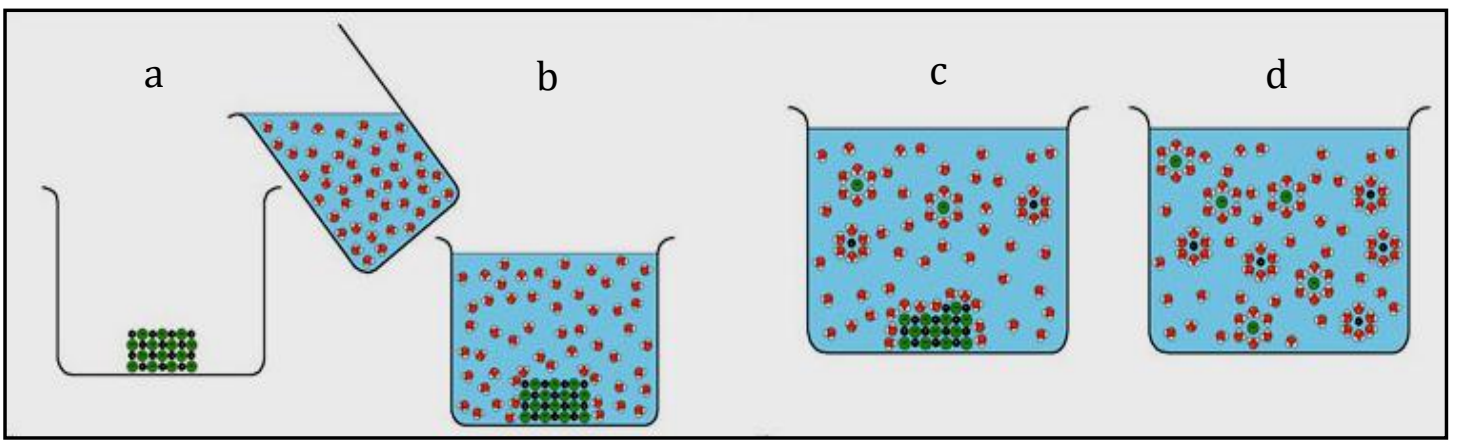

Figure 1. Représentation iconographique du modèle chimique de dissolution du chlorure de sodium dans l'eau (IUPAC, 2016)

La littérature scientifique qui traite de l'apprentissage du concept de concentration étudie principalement les difficultés liées au caractère intensif de la concentration chimique (Gandillet et Le Maréchal, 2003; Khang et Sai, 1987; Tsoumpelis et Gréa, 1995) ou celles liées aux représentations submicroscopiques (SMR) de la matière dissoute dans une solution (de Berg, 2012; Devetak, Vogrinc et Glažar, 2008).

Dans un précédent article (Willame et Snauwaert, 2015), nous avons proposé les résultats d'une étude diagnostique qui met en évidence cinq difficultés récurrentes auxquelles les apprenants sont confrontés lorsqu'ils répondent à des questions sur la concentration chimique. La première est liée au caractère intensif de la concentration chimique, une seconde à la confusion entre le volume de solvant et celui de solution et une troisième, au vocabulaire spécifique utilisé pour exprimer ce concept. Les deux autres sont plus générales aux sciences (erreurs de calculs et choix des unités).

Dans ce présent article, nous nous limitons à présenter les outils didactiques utilisés pour remédier à la confusion entre le volume de solvant et celui de la solution. Le calcul d'une concentration massique ou molaire demande de rapporter la quantité de soluté au volume total de la solution. Pour concrétiser l'erreur commise dans le calcul d'une concentration si le volume occupé par le soluté est négligé, citons cet exemple : le volume de solution après ajout de $100 \mathrm{~g}$ de chlorure de sodium (sel de cuisine) dans $500 \mathrm{~mL}$ d'eau déminéralisée est d'environ $550 \mathrm{~mL}$ à température ambiante ; l'augmentation de volume n'est pas négligeable. En prenant en compte le volume de solution, la concentration massique de chlorure de sodium n'est pas de $200 \mathrm{~g} / \mathrm{L}$ (100 g dans $500 \mathrm{~mL}$ d'eau), mais d'environ $182 \mathrm{~g} / \mathrm{L}$ (100 g dans $550 \mathrm{~mL}$ de solution). L'erreur commise sur le calcul de la concentration massique avoisine donc les $10 \%$.

\subsection{Les conceptions, le changement conceptuel et le changement de prévalence conceptuelle}

\subsubsection{Les conceptions en didactique}

Notre recherche se situe dans le cadre théorique du changement conceptuel. Nous reprenons la définition classique donnée en didactique pour la «conception» comme une construction intellectuelle du sujet qui est mobilisée spontanément face à une question ou un problème. Le sujet se réfère à des systèmes de connaissances pour résoudre une thématique qui a fait l'objet ou non d'un apprentissage (Reuter et al., 2013).

Les conceptions peuvent être exprimées explicitement par les élèves dans leurs productions. Si ce n'est pas le cas, le chercheur doit tenter de les identifier. Or les productions des apprenants sont réalisées dans un contexte particulier ; elles répondent à une question dans des conditions précises. Leur interprétation est donc liée à la fois aux conditions de la production et aussi au filtre qu'exerce le chercheur à travers ses propres cadres conceptuels et ses propres positions théoriques (« hypothèses de représentation », Astolfi, 1992).

Les conceptions des élèves peuvent être compatibles avec les connaissances scientifiques que l'école cherche à enseigner, mais elles peuvent aussi les contredire partiellement ou totalement. Christian Orange et Denise Orange-Ravachol (2013) résument les points importants concernant les apprentissages scientifiques :

L'apprentissage ne se fait pas à partir de rien : l'élève a des façons de penser les questions scientifiques et des connaissances avant enseignement, de sorte que celui-ci ne vise pas simplement à apporter des connaissances mais à changer les conceptions des élèves.

Ces conceptions ont une résistance au changement car elles sont, dans une certaine mesure, cohérentes et efficaces [...]. (p.49-50)

Selon les auteurs, il existe des divergences de vue sur l'origine des conceptions et c'est pourquoi ils proposent des chemins différents pour amener l'apprenant à se rapprocher du concept savant ou tout du moins du concept transposé pour son enseignement.

Tous ces auteurs convergent toutefois vers une idée commune : les élèves ne sont pas passifs, mais construisent leurs propres connaissances (Viennot, 2008). 


\subsubsection{La théorie classique du changement conceptuel}

Le modèle de Posner, Strike, Hewson et Gertzog

Selon George J. Posner, Kenneth A. Strike, Peter W. Hewson et William A. Gertzog (1982), l'apprentissage est pensé comme un « changement conceptuel» (« conceptual change »). Pour proposer ce nouveau paradigme en didactique, ils se basent, entre autres, sur les travaux de l'épistémologiste Thomas S. Kuhn (1983) qui compare les changements de conceptions aux « révolutions scientifiques » (vues comme des « crises ») dans l'histoire des sciences. Ils se basent également sur les travaux du suisse Jean Piaget qui définit le développement d'un individu comme un processus continu, progressif et évolutif dirigé par des nécessités internes d'équilibre. Selon Piaget, l'adaptation de l'individu à son milieu se fait par une dynamique « assimilation » et « accommodation ». L'assimilation est le processus par lequel un objet de l'environnement avec lequel l'individu est en interaction est directement intégré par la structure mentale de celui-ci. Inversement, l'accommodation est le processus par lequel la structure mentale de l'individu se modifie pour s'adapter à son environnement (Houdé, 2004, p. 10).

Ainsi, Posner et al. (1982) pensent que, tout comme le scientifique doit comprendre et expliquer de nouveaux concepts en révisant (en « révolutionnant ») sa façon de voir le monde, l'apprenant doit également s'engager à changer ses conceptions via les processus d'assimilation et d'accommodation.

[T] he scientist must acquire new concepts and a new way of seeing the world. Kuhn terms this kind of conceptual change a "scientific revolution". [...] We believe there are analogous patterns of conceptual change in learning. Sometimes students use existing concepts to deal with new phenomena. This variant of the first phase of conceptual change we call assimilation. Often, however, the students' current concepts are inadequate to allow him to grasp some new phenomenon successfully. Then the student must replace or reorganize his central concepts. This more radical form of conceptual change we call accommodation. (Posner et al., 1982, p. 212)

Selon Posner et al., le changement conceptuel ne peut avoir lieu que si quatre conditions sont réunies. La première condition posée par Posner et al. est l'«insatisfaction »dans le sens où l'apprenant doit être conscient que ses propres conceptions ne permettent plus d'expliquer un nouveau phénomène auquel il est confronté.

Une seconde condition est liée à l'«intelligibilité » des nouvelles conceptions: elles doivent être amenées à l'apprenant de telle façon qu'il soit capable de les comprendre et de se les représenter. Ces mêmes conceptions doivent être «plausibles » c'est-à-dire cohérentes par rapport au problème donné de façon à pouvoir le résoudre. Enfin, la dernière condition est que ces nouvelles conceptions soient «fécondes » c'est-à-dire efficaces pour expliquer d'autres phénomènes. Selon ce modèle, ces quatre conditions sont nécessaires pour permettre l'accommodation. Celle-ci est certes un changement radical à réaliser, mais il se fait graduellement par petits ajustements successifs.

Posner et al. précisent l'écologie du changement conceptuel : en classe, il est impératif d'avoir recours au conflit cognitif, sorte de crise scientifique comme l'entendait Kuhn, pour obtenir l'accommodation.

Jean-Pierre Astolfi, Eliane Darot, Yvette Ginsburger-Vogel et Jacques Toussaint (2008) définissent ainsi le conflit cognitif :

Un conflit cognitif se développe lorsqu'apparaît, chez un individu, une contradiction ou une incompatibilité entre ses idées, ses représentations, ses actions. Cette incompatibilité, perçue comme telle ou, au contraire, d'abord inconsciente, devient la source d'une tension qui peut jouer un rôle moteur dans l'élaboration de nouvelles structures cognitives. (p. 35)

Le modèle de Posner et al. (1982), rejoint celui de Nussbaum et Novick (1982) qui, au même moment, proposent également le conflit cognitif pour permettre le changement conceptuel. D'un point de vue pratique, ces auteurs pensent qu'il est indispensable de produire un déséquilibre chez l'apprenant, une prise de conscience parfois brutale qui lui permette de se rendre compte des limites de ses conceptions. Ils proposent trois étapes à suivre: «(1) exposing alternative frameworks, (2) creating conceptual conflict, (3) encouraging cognitive accommodation 》 (Nussbaum et Novick, 1982, p. 183).

En 1992, Strike et Posner révisent leur modèle trop centré sur les processus cognitifs de l'apprenant et ils ajustent leur idée d'écologie conceptuelle en y incorporant des composantes affectives (comme la motivation) et sociales :

This theory of conceptual change is embedded in a set of epistemological assumptions that are far more generalizable than our application to misconceptions has exploited. These epistemological assumptions suggest that the basic problem of understanding cognitive development is to understand how the components of an individual's conceptual ecology interact and develop and how the conceptual ecology interacts with experience. (Strike et Posner, 1992, p. 155-156)

De plus, les conceptions des élèves étaient initialement vues comme des objets auxquels l'écologie conceptuelle s'appliquait. Strike et Posner les reconnurent comme faisant partie de l'écologie conceptuelle. Selon eux, les conceptions sont un outil de pensée à la disposition de l'individu pour appréhender le monde et peuvent donc en déformer la perception lorsqu'elles sont erronées. Ils préconisent alors de «déraciner» les éléments de l'écologie conceptuelle qui favorisent les conceptions erronées et de les remplacer.

Les modèles «Knowledge-as-theory » et « Knowledge-in-pieces 》

Les modèles qui suivent s'orientent davantage sur l'origine des conceptions des apprenants pour mieux comprendre les processus mentaux à l'origine du changement conceptuel : les conceptions faisant partie de référentiels rigides (modèle 
«Knowledge-as-theory ») et les conceptions comme des habitudes spontanées isolées («Knowledge-in-pieces 》).

Selon Stella Vosniadou, les élèves se construisent très tôt un référentiel relativement rigide formé de présuppositions ontologiques et épistémologiques dans lequel ils chercheront par la suite à inscrire les nouveaux apprentissages (Vosniadou et Brewer, 1992). Ce référentiel serait formé d'un certain nombre de croyances ou «Cadres théoriques naïs » («Framework theory»). Un exemple de cadre théorique naif est : « Ce qui ne se voit plus, n'existe plus » (Potvin, 2011). Pour Stella Vosniadou, le changement conceptuel aura lieu si les modèles mentaux sont révisés par les élèves puis finalement remplacés par les modèles scientifiques reconnus. Pour les défenseurs de cette perspective «Knowledge-as-theory », le conflit cognitif permet le changement conceptuel.

Un autre courant propose le modèle de «Connaissances en pièces » («Knowledge-in-pieces »). Andrea diSessa (1983, 2013) en est l'un des principaux défenseurs. Selon cet auteur, les élèves ne formulent pas leurs conceptions à partir de théories bien élaborées, mais plutôt à partir d'habitudes interprétatives intuitives et élémentaires. Il nomme ces habitudes des «p-prims» ("phenomenological primitives » ou «primitives phénoménologiques »). Les élèves jugent pertinent d'utiliser une $p$-prim sur la seule base des apparences et de leurs impressions afin de produire des explications et prendre des décisions. Ruth Stavy et Dina Tirosh (2000) utilisent le terme «intuitive rules » ("règles intuitives ») pour nommer ces habitudes spontanées. Citons également les «core intuitions » («intuitions de base ») de Brown (1993).

Un exemple de $p$-prim mis en évidence par diSessa (2006) lors de l'apprentissage de la loi d'Ohm: «More effort begets more results » (p. 274). Cette habitude appelée «causalité proportionnelle » est également citée par Stavy et Tirosh (2000) sous la forme : «More A then more B » (p. 1) : « Si la valeur de A augmente alors celle de B augmente aussi ».

Selon diSessa, les $p$-prims s'accumulent jour après jour suite aux expériences de la vie, aux interactions physiques et psychologiques avec l'environnement naturel et social. Le terme «phénoménologique» (du grec: «étude de ce qui apparaît ») découle de ce que chacun voit et retient de ses expériences quotidiennes. Le mot «primitive » est donné du fait que ces habitudes sont à l'origine des conceptions comme le génotype est à l'origine du phénotype en génétique.

Les auteurs insistent aussi sur le caractère intuitif de ces habitudes dans le sens où l'individu est incapable de les expliquer puisqu'elles lui paraissent évidentes. Les mêmes habitudes interprétatives peuvent être «bonnes» dans certains contextes et «mauvaises » dans d'autres. L'expert est censé utiliser la bonne habitude dans un certain contexte, ce que ne réalise pas toujours le novice. Le contexte a d'ailleurs dans ce modèle une influence majeure, ce qui explique le caractère incohérent des réponses des élèves pour un même concept scientifique par simple modification du contexte.
Comme pour le modèle de Vosniadou, si l'enseignant ne tient pas compte de ces habitudes intuitives, l'apprenant ne pourra pas «accommoder» à long terme les nouveaux concepts dans sa structure cognitive.

Pour diSessa, un raisonnement est toujours logique, seule(s) une (des) habitude(s) pour le mener est (sont) erronée(s). Une solution est d'analyser avec l'apprenant tous les éléments de son raisonnement en cherchant à trouver ceux qui sont à l'origine de l'erreur de façon à les réviser.

Ainsi, selon cette optique, l'élève ne raisonnerait pas en référence à un cadre théorique cohérent (« Knowledge-astheory »), mais en puisant dans une collection non structurée d'éléments simples («Knowledge-in-pieces »). De ce point de vue, le changement conceptuel résulte davantage du passage d'un savoir fragmenté à un savoir structuré (par ajout, suppression, révision des éléments qui constituent le répertoire de $p$-prims afin de développer une classe de coordination plus conforme au savoir scientifique) plutôt que d'une modification profonde de son cadre théorique de pensée.

D'autres auteurs proposent des variantes de ces modèles comme les «conceptions d'ancrage » (Clement, Brown et Zietsman, 1989), les conceptions faisant partie d'une 《structure conceptuelle» (Duit et Treagust, 2003), le concept de «facettes », selon lequel une classification des idées spontanées des apprenants est réalisée (Minstrell, 2001).

Nous ne développons pas ces modèles, dont la liste n'est d'ailleurs pas exhaustive. Les lunettes à travers lesquelles nous étudions les conceptions des apprenants dans la suite de notre texte sont celles fournies par le modèle de diSessa. Une étude diagnostique sur les difficultés d'acquisition du concept de concentration a été réalisée dans la phase qui précède celle dont traite ce présent article. Elle nous a permis de mettre en évidence le caractère intuitif des réponses données par les élèves interrogés sur le volume de solution, comme des «réflexes » mobilisés sans raisonnement structuré. Les élèves interrogés semblaient utiliser une heuristique qui leur permettait de répondre rapidement à la question sans appui sur une théorie élaborée et rigide. Une modification mineure de l'énoncé, sans conséquence sur le raisonnement à utiliser ni sur le résultat, les amenait à utiliser une heuristique parfois opposée (Willame et Snauwaert, 2015).

2.2.3 L'apport des neurosciences cognitives au modèle du changement conceptuel

L'utilisation des techniques d'imagerie fonctionnelle cérébrale a permis d'étudier les régions du cerveau qui s'activent lors de la réalisation de tâches cognitives.

Jonathan A. Fugelsang et Kevin N. Dunbar font partie des premiers chercheurs en didactique à se tourner vers l'utilisation de l'imagerie cérébrale. Ils constatent en effet la difficulté à obtenir un changement conceptuel. Ils ont voulu 
établir un lien entre le conflit cognitif et les régions du cerveau qui y sont associées. Leur approche est d'examiner le recrutement des circuits neurologiques cérébraux qui sont impliqués lorsque les individus reçoivent des données incohérentes avec leurs propres conceptions. Leurs résultats d'expériences publiées en 2005 (Fugelsang et Dunbar, 2005) montrent que lorsqu'un sujet est mis en situation de conflit cognitif, il traite l'information comme une erreur et il détourne son attention. Au final, le sujet n'assimile pas la nouvelle conception proposée. Selon Dunbar, cette expérience démontre l'une des raisons pour laquelle le changement conceptuel soit difficile à obtenir.

Plutôt que de comparer l'activité cérébrale avant et après la réalisation d'un changement conceptuel, une stratégie d'étude consiste à comparer l'activité cérébrale d'un groupe d'experts qui semblent avoir réalisé un changement conceptuel avec un groupe de novices ne l'ayant pas réalisé. C'est de cette façon que Dunbar, Fugelsang et Stein procèdent à de nouvelles expérimentations publiées en 2007. Ils constatent que les conceptions erronées sont toujours présentes même chez un expert mais que celui-ci est capable d'activer certaines zones cérébrales pour, semble-t-il, inhiber les théories naïves et donc proposer une réponse correcte :

Yet the results of the experiments reported in this chapter indicate that even when conceptual change appears to have taken place, students still have access to the old naïve theories and that these theories appear to be actively inhibited rather than reorganized and absorbed into the new theory. (Dunbar, Fugelsang et Stein, 2007, p. 202)

Steve Masson en 2012 arrive aux mêmes conclusions. Il observe que les experts possèdent toujours la trace de leurs fausses conceptions, mais qu'ils les inhibent, ce qui leur permet de résoudre scientifiquement des problèmes :

Plus précisément, notre interprétation des résultats obtenus appuie l'idée selon laquelle les experts possèderaient toujours dans leur cerveau la trace d'une conception inappropriée [...] et que, suite à leur formation scientifique, ils auraient développé une conception plus conforme aux savoirs scientifiques, mais n'auraient pas effacé de leur cerveau leur conception initiale qu'ils doivent alors inhiber. (Masson, 2012, p. 111)

Déjà en 1999, Olivier Houdé étudie le comportement de jeunes enfants et il envisage que le développement de l'intelligence ne consiste pas seulement à construire et à activer des stratégies cognitives nouvelles, comme le pensait Jean Piaget. L'enfant doit aussi apprendre à bloquer, inhiber la stratégie inadéquate. En parlant de la tâche de la conservation du nombre, Houdé explique :

Cette tâche a été reprise de façon informatisée en IRMf avec des enfants d'école maternelle et élémentaire, révélant qu'elle mobilisait non seulement les régions du cerveau dédiées au nombre (le cortex pariétal), mais aussi les régions du cortex préfrontal dédiées à l'inhibition des automatismes : ici l'automatisme selon lequel en général la longueur varie avec le nombre. Cela amène à réviser la théorie de Piaget en y ajoutant le rôle clé de l'inhibition cognitive comme mécanisme positif du développement de l'intelligence de l'enfant. (Houdé, 2016a, p. 21)

Selon Houdé, le modèle le plus adapté pour expliquer le développement cognitif semble le modèle de « vagues qui se chevauchent 》 (《 overlapping waves ») proposé par Robert S. Siegler (1996): au cours de son développement, l'enfant accumule un réservoir de stratégies où il puise celle qu'il juge la plus efficace dans le contexte où il se trouve. Houdé et son équipe traduisent ce modèle en y ajoutant une composante liée à l'inhibition. L'individu met au point et accumule des heuristiques qui lui permettent de réagir rapidement à diverses situations. Chaque stratégie ayant son propre champ d'application, l'individu est donc amené à inhiber l'une des stratégies et à en activer une autre afin de répondre au mieux à une situation donnée. Si l'inhibition n'est pas correctement effectuée, la réponse à une question impliquant une conception erronée sera erronée.

Lors du processus de contrôle inhibiteur lié au raisonnement conditionnel, Houdé et al. (2000) expliquent :

We demonstrate how a set of left-prefrontal regions recruited by the executive component of object, spatial, and verbal working memory, and a rightprefrontal region ensuring the emotionalmotivational aspects of decision making, all work together in a dynamic way to inhibit a perceptual bias and activate a deductive reasoning process. (p. 725)

Avec l'utilisation de l'imagerie cérébrale, Houdé et TzourioMazoyer (2003) montrent qu'un apprentissage à l'inhibition permet une reconfiguration des zones d'activation cérébrale qui se sont déplacées de la partie postérieure du cerveau à sa partie antérieure (au cortex préfrontal).

Le modèle de prévalence conceptuelle (Potvin, 2013 ; Potvin, Sauriol et Riopel, 2015) tente un ajustement de la théorie classique du changement conceptuel par l'apport des études en neurosciences et psychologie cognitives et il propose des conditions pour l'apprentissage en classe. Selon ce modèle, l'apprentissage ne se réalise qu'en situation de compétition entre différentes conceptions. Ainsi, il ne faut pas produire de conflit cognitif chez l'apprenant tant qu'il n'accorde pas de crédit à une autre stratégie possible. Dans un deuxième temps, lorsque l'apprenant est conscient des insuffisances de certaines conceptions dans certains contextes, il doit installer des signaux d'alerte inhibitifs («inhibitive stop signs ») pour bloquer leur utilisation. L'apprenant doit ensuite automatiser le procédé d'inhibition de la (des) conception(s) non pertinente(s) dans le contexte de la question par un 
entrainement métacognitif à l'inhibition. Dans le modèle de prévalence conceptuelle, le rôle du conflit cognitif est donc envisagé différemment :

[...] le conflit cognitif aurait d'abord et avant tout pour but de rendre explicite la conception initiale de manière à permettre qu'on développe éventuellement une bonne capacité à la reconnaître distinctement lorsqu'elle se manifeste, ainsi que celle de pouvoir choisir de refuser, consciemment, puis éventuellement de manière automatisée, de la mobiliser. (Potvin, 2011, p. 218).

Ainsi, les recherches en neurosciences ont permis de proposer un nouveau modèle : il ne s'agirait pas de changer les conceptions, autrement dit «d'effacer» ou de restructurer de façon approfondie les réseaux neuronaux en place, mais plutôt d'apprendre à « inhiber » ceux menant à la formulation de réponses inappropriées et d'activer ceux conduisant à la conception experte (Babai, Younis et Stavy, 2014 ; Brault Foisy et al., 2015 ; Houdé, 2016a ; Masson, 2012 ; Potvin, Riopel et Masson, 2007).

Plusieurs études rapportent l'importance du contrôle inhibiteur comme fonction exécutive dans l'acquisition de connaissances en mathématiques, orthographe ou lors de l'exécution de consignes (Borst, Moutier et Houdé, 2014 ; Cassotti et Moutier, 2010 ; Lubin et al., 2012 ; Rossi et al., 2012). Le sujet doit apprendre à bloquer certaines réponses intuitives erronées dans le contexte de la question pour progresser dans l'apprentissage du concept envisagé. L'élève doit s'entrainer à corriger ses erreurs à l'aide d'alertes et, pour ce faire, il manipule un dispositif tel qu'un «attrape-piège ». Rossi et al. (2012) décrivent ainsi leur attrape-piège :

Il se matérialise par une planche sur laquelle est apposé un transparent que l'enfant peut soulever et replacer à sa guise. Sur ce transparent apparaît une zone hachurée qui matérialise les processus attentionnels d'inhibition, sous laquelle l'enfant va être amené à placer la réponse erronée. Ce transparent est troué en son centre laissant apparaitre une zone d'activation attentionnelle non hachurée dans laquelle l'enfant va être amené à placer la réponse correcte. Les cartons réponses, d'autres alertes visuo-spatiales, sont colorés en rouge ou vert selon qu'ils représentent la mauvaise ou la bonne réponse. (p. 40).

Les résultats de ces études encouragent à développer l'apprentissage à l'inhibition en classe.

\section{Questions de recherche}

\subsection{Origine des questions de recherche}

Une des phases de notre recherche a consisté à comprendre l'origine de la non considération de l'augmentation de volume de la solution lorsqu'un soluté est dissout dans un solvant. Pour ce faire, une première étape a été de poser une question simple à 135 élèves de différents niveaux scolaires (tous issus du système scolaire belge francophone) : «Que fait le niveau de liquide si du sel de cuisine est ajouté puis dissout (après agitation) dans un volume d'eau? ». La question était accompagnée d'un support matériel: un tube à essais contenant de l'eau et un second avec quelques grammes de sel. Les prévisions des interrogés sont proposées dans le tableau 1.

Ainsi, 91 \% des élèves de première primaire, non experts en chimie, répondent correctement à cette question contre $37,5 \%$ des élèves de quatrième secondaire et $34,5 \%$ des futurs enseignants de sciences.

Dans une deuxième étape, des entretiens d'explicitation (Vermersch, 2011) ont permis de mettre en évidence que la plupart des enfants de 6-7 ans utilisent la stratégie «Plus de matière donc plus de volume »; stratégie que nous associons à la $p$-prim de causalité proportionnelle «More $\mathrm{A}$ then more $\mathrm{B}$ 》.

La majorité des élèves plus âgés qui ont suivi des enseignements en chimie n'utilisent plus cette stratégie pourtant efficace dans le contexte de la dissolution d'un soluté dans un solvant et donc du calcul d'une concentration chimique.

Tableau 1. Prévisions selon la classe des interrogés à la question «Que fait le niveau de liquide si du sel de cuisine est ajouté puis dissout (après agitation) dans un volume d'eau? »

\begin{tabular}{|c|c|c|c|c|}
\hline \multirow[b]{3}{*}{ Classe des interrogés } & & & & \\
\hline & \multicolumn{4}{|c|}{ Prévisions } \\
\hline & Effectif & $\begin{array}{c}\text { Le niveau reste le } \\
\text { même }\end{array}$ & $\begin{array}{l}\text { Le niveau monte } \\
\text { (réponse correcte) }\end{array}$ & Le niveau baisse \\
\hline $1^{\text {ère }}$ primaire (6-7 ans) & 36 & $3 \%$ & $91 \%$ & $6 \%$ \\
\hline $6^{\mathrm{e}}$ primaire (11-12 ans) & 30 & $40 \%$ & $47 \%$ & $13 \%$ \\
\hline $4^{\mathrm{e}}$ secondaire (15-16 ans) & 40 & $60 \%$ & $37,5 \%$ & $2,5 \%$ \\
\hline $\begin{array}{l}\text { Futurs enseignants de sciences } \\
\text { ( } 6 \text { ans d'études supérieures en sciences) }\end{array}$ & 29 & $62 \%$ & $34,5 \%$ & $3,5 \%$ \\
\hline
\end{tabular}


Les explications données par les élèves de 15-16 ans (grade 10) qui répondent de façon incorrecte que le niveau reste le même sont probablement liées à des utilisations ou interprétations erronées des modèles chimiques et de leurs représentations iconographiques. Selon de Berg (2012), la visualisation du modèle de particules de la matière, qui est déterminante pour comprendre le concept de concentration, est connue pour présenter des difficultés pour les étudiants. Selon lui, les locutions verbales associées aux modèles chimiques ne permettent pas à tous les apprenants en chimie de déclencher une image moléculaire visuelle d'où l'utilisation des représentations submicroscopiques (SMR pour 《submicroscopic representations »). Mais ces visualisations du modèle particulaire de la matière peuvent elles-mêmes poser des difficultés aux apprenants. De même, Tümay (2016) ajoute qu'une autre difficulté dans l'apprentissage de la chimie est liée à la compréhension des interactions entre les entités qui constituent la matière. Celles-ci dépendent de nombreux paramètres et les élèves ont des difficultés à considérer plusieurs données microscopiques ensemble pour comprendre les modèles proposés par les experts.

Citons quelques exemples de stratégies que les élèves interrogés ont explicitées et qui seraient à l'origine de la non considération de la place occupée par le soluté dissout :

\section{1. « car le sel se dissout ».}

Le fait de se dissoudre est une justification en soi. La dissolution permet d'obtenir un mélange homogène. Pour ces élèves, cela signifie que le soluté « disparait » et ils semblent en déduire qu'il n'existe plus. Le volume de soluté serait donc négligé et le volume de solvant est dès lors considéré comme le volume de solution. Ces élèves restent sur une vision macroscopique de la solution.

\section{2. «car le sel absorbe l'eau ».}

Cette réponse est peut-être liée à des expériences de la vie (scolaire ou non) où des poudres «pompent» des liquides sans qu'un volume supplémentaire ne paraisse occupé. Tout comme les élèves du point précédent, leur raisonnement se base sur une vision macroscopique de la matière: ils n'envisagent pas les entités de soluté comme des entités individuelles capables de réaliser des liaisons avec l'eau. Ils s'en tiennent à la matière «sel » en tant qu'« ensemble ».

3. «car les entités de sel vont combler l'espace entre les molécules d'eau ».

Pour les élèves qui utilisent cette stratégie de réponse, la vision microscopique de la solution détermine leur raisonnement puisqu'ils envisagent, à l'inverse des élèves précédents, la solution comme un ensemble d'entités bien distinctes : les molécules d'eau et les entités de soluté. Pour eux, le soluté ne disparait pas, mais il prend la place qui existe entre les molécules d'eau. Pour ces élèves, il est possible que les représentations iconographiques des molécules d'eau en forme de ronds mènent à cette conception. Cette stratégie est logique et en partie vraie puisque le volume de sel avant dissolution additionné au volume d'eau de départ est supérieur au volume de solution une fois le sel dissout dans l'eau.

Pour les futurs enseignants de sciences qui répondent majoritairement de façon incorrecte que le niveau de liquide reste le même, les explications sont extrêmement variées. Les nombreuses justifications différentes proposées par les étudiants universitaires interrogés ont toutefois un point commun : elles utilisent un langage issu de la chimie et se réfèrent à de multiples modèles chimiques. Les étudiants s'expriment en utilisant les termes «interactions entre les ions sodium et chlorure », «solvatation des ions présents », 《polarité de la molécule d'eau », « saturation de l'eau en sel », 《 réactions chimiques », «masse volumique », etc.

Deux précédentes publications développent les différentes conceptions des différents groupes d'interrogés (Willame et Snauwaert, 2016 ; Willame, 2017).

\subsection{Nos questions de recherche}

Pour utiliser correctement le concept de concentration chimique, l'apprenant doit prendre conscience que le volume de solution est supérieur au volume de solvant. Il doit activer (ou réactiver) cette $p$-prim: «Plus de matière donc plus de volume » dans le contexte de calculs de la concentration d'un soluté au sein d'une solution. Pour ce faire, des outils didactiques et neuroscientifiques utilisant le conflit cognitif et l'entrainement à la fonction exécutive de contrôle inhibiteur des conceptions non pertinentes doivent être construits, testés et confrontés. Notre objectif est d'améliorer les scores obtenus par les élèves interrogés dans ce contexte.

Trois questions de recherche ont été posées :

- Les scores des apprenants sont-ils améliorés après un conflit cognitif?

- Les scores des apprenants sont-ils améliorés si le conflit cognitif est suivi d'un entrainement à l'inhibition des conceptions non pertinentes?

- Quel que soit le type de question posée à un apprenant, l'entrainement à l'inhibition a-t-il le même effet sur la réponse finale?

\section{Méthodologie}

\subsection{Participants}

Notre étude a été réalisée dans deux écoles de Belgique francophone entre mars 2015 et mars 2016. Huit classes de grade 10 (élèves entre 15 et 17 ans) ont participé à la recherche pour un total de 150 élèves. Tous les élèves étaient issus de classes mixtes ayant un cours de 5 heures par semaine en sciences (classes dites « en option sciences ») dont 2 heures de chimie. Ils avaient tous suivi, dans l'année, l'apprentissage du concept de concentration chimique. 
Quatre classes ont suivi l'activité impliquant un conflit cognitif seul soient 72 élèves (groupe CC pour «Conflit Cognitif ») et quatre classes ont suivi l'activité impliquant un conflit cognitif suivi d'un entrainement à la fonction exécutive de contrôle inhibiteur des conceptions non pertinentes soient 78 élèves (groupe $\mathrm{CC}+\mathrm{EI}$ pour «Conflit Cognitif » et « Entrainement à l'Inhibition »).

\subsection{Chronologie de l'expérimentation et conditions de passation}

Chaque élève a été soumis à un pré-test comprenant quatre questions à propositions multiples et choix unique (annexe 1). Pour répondre correctement à ces questions sur la concentration chimique, l'interrogé doit avoir pris conscience que le volume de solution est supérieur au volume de solvant et que pour calculer une concentration, le volume de solution doit être utilisé. Chaque groupe a ensuite été soumis à une activité : le groupe $\mathrm{CC}$ a été soumis à une activité impliquant un conflit cognitif et le groupe CC+EI a été soumis à une activité impliquant un conflit cognitif suivi d'un entrainement à linhibition des conceptions non pertinentes dans le contexte de la concentration chimique. Chaque élève a ensuite répondu à un post-test (quatre questions similaires à celles du pré-test). Le post-test a été proposé juste après l'activité.

Ces activités se veulent utilisables en situation de classes réelles et leur durée de réalisation ne dépasse pas une période de cours soit 50 minutes. Chacune des étapes de l'expérimentation était décrite sur des feuilles séparées et fournies au fur et à mesure que l'élève avait fini une étape. L'élève était seul pour répondre aux différentes étapes sans possibilité de communiquer avec ses pairs.

\subsection{Outil visant le conflit cognitif}

Lors de la phase diagnostique, notre recherche a montré que de nombreux élèves considèrent le volume de solvant et non de solution pour le calcul de la concentration en soluté. Les entretiens d'explicitation ont mis en évidence que ces apprenants n'envisagent pas la place supplémentaire occupée par le soluté. L'activité construite (annexe 2) s'appuie sur ces résultats. Elle fait appel à une manipulation simple: deux tubes à essais sont présentés à l'élève. Le premier contient 20 millilitres d'eau (niveau d'eau marqué par un trait sur le tube) et le second contient 3 grammes de sel de cuisine (chlorure de sodium). Il est demandé à l'apprenant de prévoir ce que va devenir le niveau de solution suite à la dissolution du sel dans l'eau. L'élève réalise ensuite la manipulation et confronte sa prévision à son observation. Le niveau de liquide augmente de façon très visible (élévation du niveau de liquide dans le tube de $0,5 \mathrm{~cm}$ ). Si l'élève observe une différence, un conflit perceptivo-cognitif devrait se réaliser.

Suite à cette activité, afin d'institutionnaliser l'importance du volume de solution dans le calcul d'une concentration, un texte est donné à chaque élève. Il lui est rappelé que :

1. le volume de solution est supérieur au volume de solvant ;
2. pour calculer la concentration d'un soluté, il faut considérer le volume de solution et pas celui de solvant ;

3. si le volume total de la solution n'est pas connu, il est impossible de calculer la concentration du soluté.

4.4 Outil visant l'entrainement à la fonction exécutive de contrôle inhibiteur des conceptions non pertinentes

L'activité doit permettre de réactiver la prévalence d'une habitude intuitive que la plupart des apprenants utilisent spontanément lorsqu'ils sont encore en première primaire à savoir « More A then more B ». Elle n'est plus prévalente dans le contexte de la dissolution d'un sel dans l'eau au fur et à mesure que les apprenants approfondissent les modèles chimiques utilisant les représentations symboliques des constituants de la matière et de leurs interactions (tableau 1).

L'activité amenant l'élève à s'entrainer à inhiber les conceptions non pertinentes dans le contexte de la dissolution d'un soluté dans un solvant et du calcul d'une concentration chimique consiste, dans un premier temps, à faire prendre conscience à l'apprenant de la compétition entre deux conceptions :

- Conception «Vsolution» (pertinente dans les contextes de la dissolution d'un soluté dans un solvant et du calcul d'une concentration chimique) : «Après dissolution d'un soluté dans un solvant, le volume de solution est supérieur au volume de solvant »,

- Conception «Vsolvant» (non pertinente dans les mêmes contextes) : «Après dissolution d'un soluté dans un solvant, le volume de solution est égal ou inférieur au volume de solvant ».

L'élève est alors invité à activer celle pertinente dans le contexte du calcul d'une concentration (conception «Vsolution») et mis en garde de ne pas employer la conception non adéquate (conception «Vsolvant »).

Dans un deuxième temps, l'élève de grade 10 est amené à automatiser l'activation de la conception pertinente «Vsolution » et l'inhibition de la conception non pertinente «Vsolvant ». Nous avions opté pour un dispositif d'« attrapepiège » fait d'un cache avec fenêtre, d'un carton rouge (conception à inhiber: «Je calcule la concentration en utilisant le volume de solvant») et d'un carton vert (conception à activer: «Je calcule la concentration en utilisant le volume de solution ») de Rossi et al. (2012). Suite aux premiers essais, nous avons constaté que les adolescents de grade 10 interrogés ne suivaient pas la consigne de répéter l'opération pour chacune des questions posées. L'automatisation du processus d'inhibition n'a donc pas été réalisée. Cette constatation a également été faite avec des élèves de grade 9 lors d'un master en sciences de l'éducation (Van Lerberghe, 2017).

Nous avons préféré construire un autre dispositif qui conviendrait davantage à l'âge des élèves ciblés par notre étude (adolescents de 16-17 ans) dont l'utilisation serait 
inévitable à chaque question posée et où l'élève en remédiation pourrait être lui-même un « attrape-piège ».

L'élève de grade 10 est invité à vérifier la copie d'un élève de grade 9, prénommé Alex, qui a écrit cinq affirmations en lien avec le volume considéré dans le calcul d'une concentration. L'élève de grade 10 en activité doit contrôler, pour chaque phrase écrite par Alex, que celui-ci a bien activé la conception pertinente «Vsolution » pour le calcul d'une concentration et inhibé la conception non pertinente «Vsolvant». De cette façon, l'adolescent de grade 10 est responsabilisé à vérifier que l'un de ses pairs progresse correctement dans son apprentissage.

La figure 2 donne une partie du tableau que l'élève en activité doit compléter (tableau complet en annexe 3).

Les trois premières des cinq phrases sont suivies d'une correction. Les première et troisième phrases sont erronées ; le volume considéré n'est pas celui de la solution mais celui du solvant. Il ne faut pas que l'élève en remédiation commette lui aussi les mêmes erreurs qu'Alex et il doit donc vérifier que l'examen qu'il fait des phrases d'Alex est correct. Si l'élève en activité a mal corrigé Alex, il est mis en garde pour la suite : «Si tu as mal corrigé Alex, reste vigilant pour la suite : active bien la conception : la concentration se ramène au volume de solution. ».

\begin{tabular}{|l|l|l|}
\hline \multicolumn{1}{|c|}{ Phrases écrites par Alex } & $\begin{array}{c}\text { Activation de } \\
\text { Vsolution } \\
\text { Bien! }\end{array}$ & $\begin{array}{c}\text { Non activation de } \\
\text { Vsolution } \\
\text { Pas bien! }\end{array}$ \\
\hline $\begin{array}{l}\text { 1. La concentration en sel est le } \\
\text { rapport entre la quantité de sel et le } \\
\text { volume d'eau. }\end{array}$ & & \\
\hline $\begin{array}{l}\text { 2. Si je mets du sucre dans de l'eau, } \\
\text { je calcule la concentration en sucre } \\
\text { en divisant la quantité de sucre par } \\
\text { le volume d'eau sucrée. }\end{array}$ & & \\
\hline $\begin{array}{l}\text { 3. Pour préparer 1 litre d'une } \\
\text { solution de sel dont la concentration } \\
\text { sera 120 g/L, je dois mettre 1 litre } \\
\text { d'eau dans une fiole de 1 litre puis } \\
\text { rajouter 120 g de sel. }\end{array}$ & & \\
\hline
\end{tabular}

Figure 2. Partie du tableau que l'élève de grade 10 doit compléter lors de l'activité d'entrainement à l'inhibition de la conception non pertinente «Vsolvant »

Puis il poursuit avec les deux dernières phrases où la quatrième est erronée (une correction suit également ces deux phrases).

Nous avons donné un prénom à cet élève imaginaire (Alex) et associé une photo d'un jeune en âge d'être en grade 9 dans le but de motiver et d'impliquer davantage l'élève en remédiation. Le prénom «Alex » a été choisi, car il est court et il correspond à un prénom raccourci donné par les amis à une personne qui leur est proche. Ceci pour renforcer l'affectivité.
Nous nous basons sur les études des équipes de Damasio (1994), Smith et Lazarus (1990) et Houdé et al. (2001) qui ont mis en évidence que les émotions jouent un rôle biologique dans le raisonnement et la prise de décision. Smith et Lazarus précisent que l'émotion dépend d'une combinaison entre motivation, intérêt et environnement, induisant l'individualisation de la décision. Nous pensons donc que notre outil faisant appel à l'émotion devrait permettre un apprentissage optimisé.

\subsection{Analyses des données}

Les résultats des pré- et post-tests permettent d'établir des scores en fonction du nombre de réponses correctes. Nous appelons «gain conceptuel », la variation des scores entre les pré- et post-tests.

Les résultats sont analysés selon deux aspects: le gain conceptuel par élève et le gain conceptuel par question.

Gain conceptuel par élève : pour chaque élève ayant répondu aux 4 questions des pré- et post-tests, un gain conceptuel, compris entre -4 et +4 a été calculé $:$ un gain de +4 correspond à 4 réponses incorrectes au pré-test qui deviennent toutes correctes au post-test. Un 《gain 》 de -4 correspond à 4 réponses correctes au pré-test qui deviennent toutes incorrectes au post-test. Une valeur intermédiaire telle que +2 est calculée si l'élève qui répondait correctement à une question au pré-test répond correctement à trois questions au post-test. Une moyenne du gain pour l'ensemble des élèves a ensuite été calculée.

Gain conceptuel par question: pour chacune des quatre questions des pré- et post-tests et pour l'ensemble des élèves, la fréquence de réponses erronées avant l'activité qui deviennent correctes après l'activité a été calculée. Le gain conceptuel par question correspond à cette fréquence en pourcentage.

Une analyse statistique a permis de déterminer si les gains sont significatifs: distribution $t$ de Student pour le gain conceptuel par élève et distribution $\chi^{2}$ de Pearson pour le gain conceptuel par question.

\section{Résultats}

\subsection{Les conflits cognitifs apparents réalisés}

L'outil visant le conflit cognitif consiste à demander à lélève de prévoir ce que fera le niveau de liquide après l'ajout d'une quantité visible de sel dans un tube à essais contenant de l'eau, dont le niveau est marqué par un trait. Lorsque le niveau prévu par l'élève avant la manipulation est différent de ce qu'il observe après avoir réalisé la manipulation (prévision incorrecte), un conflit percepto-cognitif doit avoir lieu.

Sur base des explications de l'élève, écrites avant et après la manipulation, nous avons déterminé si un conflit cognitif 
apparent a eu lieu ${ }^{2}$. Nous préférons ajouter le terme «apparent », car nous ne pouvons pas nous placer dans la tête de l'apprenant. Nous déduisons un éventuel conflit cognitif uniquement sur la base de ce que l'élève écrit.

Nous donnons la dénomination :

« Conflit cognitif positif » («CC+ ») si l'explication de l'élève est en adéquation avec une conception experte. Nous avons attribué un CC+ aux explications où l'apprenant admet que « le sel prend de la place » ou s'il admet que «plus de matière amène plus de volume » (utilisation pertinente de la $p$-prim «More A then more B »).

Par exemple, un élève écrit « Les molécules de sel prennent de la place donc le niveau de la solution augmente » : il se rend compte que le niveau monte et justifie correctement que le sel prend de la place. Sa conception semble avoir évolué vers une conception plus experte et nous lui attribuons un conflit cognitif positif.

« Conflit cognitif négatif » («CC-») si l'explication de l'élève n'est pas en adéquation avec une conception experte.
Par exemple, un élève trouve une explication à l'augmentation du niveau de liquide en écrivant «Je pense que le liquide monte car le sel était en trop grande quantité et n'a pu se dissoudre dans l'eau ». Cet élève ne semble pas concevoir que le sel dissout occupe de la place.

«Conflit cognitif non explicite » («CC? ») si l'explication de l'élève ne permet pas de déduire si le conflit cognitif est positif ou négatif.

Un élève écrit: «Le sel s'est dissout mais a augmenté le niveau de l'eau. Je ne sais pas comment. » Il se rend compte que le niveau monte mais ne trouve pas d'explication. Un conflit cognitif a sans doute eu lieu, mais a-t-il permis le changement de prévalence, même momentané, de sa conception initiale? Nous n'en sommes pas sûrs d'où l'attribution d'un conflit cognitif non explicite.

Le tableau 2 présente, pour l'ensemble des deux groupes ayant réalisé l'activité impliquant un conflit cognitif $(n=150)$, le nombre et la fréquence de prévisions incorrectes ainsi que le nombre de conflits cognitifs (CC) positifs, négatifs et non explicites.

Tableau 2. Prévisions incorrectes et conflits cognitifs pour l'activité visant le conflit cognitif

\begin{tabular}{lccc}
\hline$n=150$ & \multicolumn{2}{c}{ Le niveau reste le même } & Le niveau diminue \\
\hline Nombre de prévisions incorrectes & $64 / 150$ & $43 \%$ & $2 / 150$ \\
Nombre de CC positifs (CC+) & $46 / 64$ & $72 \%$ & $1,5 \%$ \\
Nombre de CC négatifs (CC-) & $7 / 64$ & $11 \%$ & - \\
Nombre de CC non explicites (CC ?) & $11 / 64$ & $17 \%$ & - \\
\hline
\end{tabular}

\subsection{Gain par élève}

Sur base des quatre questions des pré- et post-tests, une moyenne du gain conceptuel pour l'ensemble du groupe a été calculée et indiquée dans le tableau 3 (un interrogé du groupe $\mathrm{CC}+\mathrm{EI}$ n'a pas répondu à deux questions au post-test; la moyenne du gain a été calculée pour un effectif de 77).

Pour le groupe $\mathrm{CC}$ soumis uniquement à un conflit cognitif, le gain conceptuel est de $+0,64$ (écart-type $=1,23$ ). L'augmentation des scores est statistiquement significative ; $t(71)=4,45, p<0,0001$.

Dans le groupe CC+EI soumis à un conflit cognitif suivi d'un entrainement à l'inhibition, le gain conceptuel $(\mathrm{M}=+1,65$, écart-type $=1,16)$ est significativement plus important que pour le groupe $\mathrm{CC} \quad(\mathrm{M}=+0,64$, écarttype $=1,23) ; t(76)=5,2, p<0,0001, d=0,85$.
Tableau 3. Confrontation des moyennes du gain conceptuel par élève pour les groupes $\mathrm{CC}$ et $\mathrm{CC}+\mathrm{EI}$

\begin{tabular}{cc}
\hline & Activité avec conflit \\
Activité & cognitif et entrainement \\
avec conflit & à l'inhibition des \\
cognitif seul & $\begin{array}{c}\text { conceptions non } \\
\text { pertinentes }\end{array}$ \\
&
\end{tabular}

\begin{tabular}{lcc} 
Groupe d'élèves & CC & CC+EI \\
Effectif $n$ & 72 & 77 \\
$\begin{array}{l}\text { Moyenne du gain } \\
\text { conceptuel par élève } \\
\text { (entre - } 4 \text { et }+4)\end{array}$ & $+0,64$ & $+1,65$ \\
$\begin{array}{l}\text { Ecart type } \\
d \text { de Cohen }\end{array}$ & 1,23 & 1,16 \\
& & 0,85 (effet fort) \\
\hline
\end{tabular}

${ }^{2}$ Les élèves ayant prévu correctement le niveau de liquide ne devaient pas expliciter leur conception après la manipulation. 
Ajoutons que 14 des 72 élèves du groupe CC ont un gain négatif (12 élèves répondent moins bien à une question après le posttest et 2 élèves à deux questions) alors qu'un seul élève des 77 du groupe $\mathrm{CC}+\mathrm{EI}$ obtient un gain négatif de -1 : cet élève avait 3 réponses erronées au pré-test et donne une réponse erronée supplémentaire au post-test (question $1 \mathrm{du}$ test).

Nous devons cependant rester prudents quant aux résultats. Une part de l'augmentation des scores est peut-être due à la durée supplémentaire que les élèves ont consacrée à la correction des affirmations d'Alex, soit environ 5 à 10 minutes selon les élèves, ainsi qu'à la pratique qu'ils en ont eue.

\subsection{Gain par question}

Nous appelons gain conceptuel par question, la fréquence en pourcentage des réponses erronées avant l'activité qui deviennent correctes après l'activité. Ainsi à la question 1 pour le groupe $\mathrm{CC}(n=72)$, nous obtenons 34 réponses erronées avant l'activité dont 25 deviennent correctes après l'activité, soit un gain conceptuel de 73,5\%.

Les résultats obtenus pour chacune des quatre questions et pour les deux groupes CC $(n=72)$ et CC+EI $(n=78)$ sont présentés dans le tableau 4.

Le graphique 1 permet de visualiser les différences de gain conceptuel par question en fonction des activités $\mathrm{CC}$ et CC+EI. Le groupe ayant suivi l'activité avec conflit cognitif et entrainement à l'inhibition obtient un gain conceptuel significativement supérieur uniquement pour les questions Q3 et Q4, par rapport au groupe ayant suivi l'activité avec conflit cognitif seul.

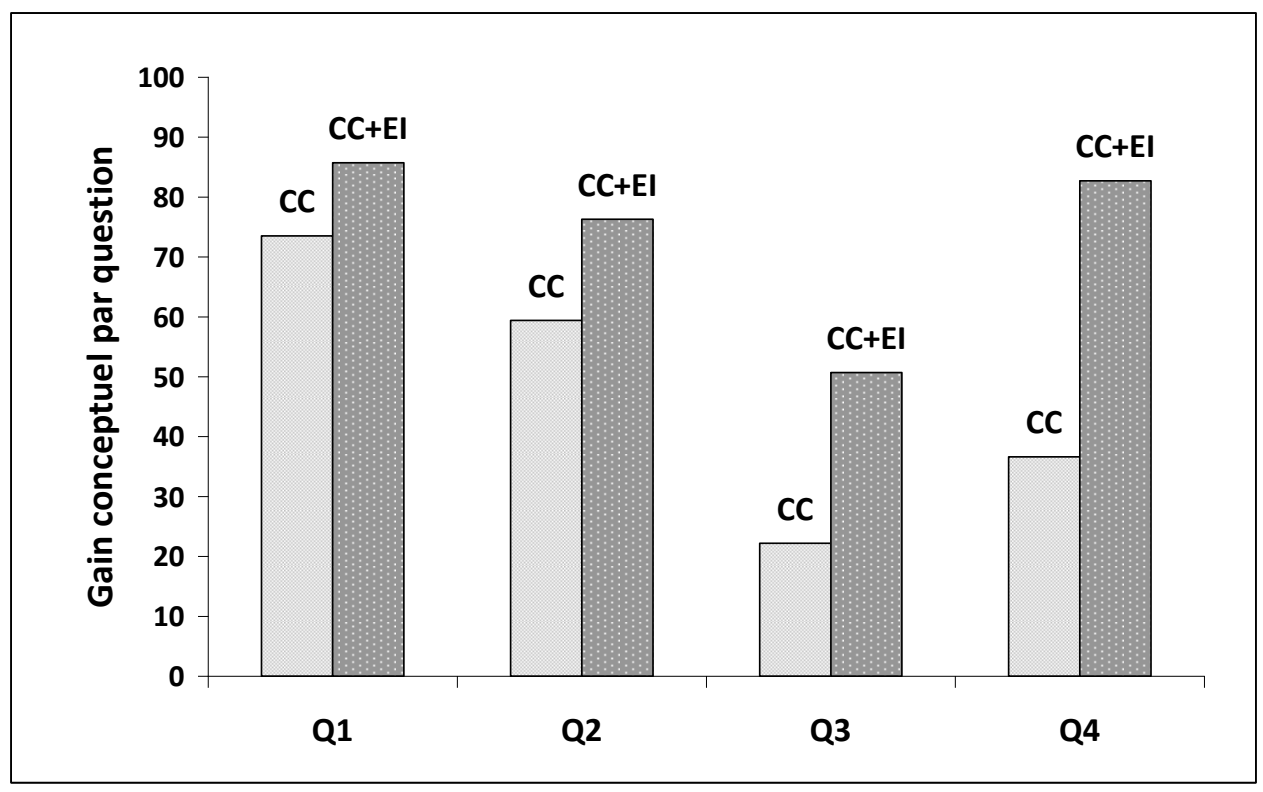

Graphique 1. Confrontation des gains conceptuels par question pour les activités CC et CC+EI

Tableau 4. Fréquence des réponses erronées avant l'activité, fréquence des réponses qui deviennent correctes après l'activité, gain conceptuel par question et tests statistiques selon la distribution de Pearson

\begin{tabular}{|c|c|c|c|c|c|c|c|c|}
\hline \multirow[t]{2}{*}{ Questions } & \multicolumn{2}{|c|}{$\begin{array}{l}\text { Fréquence des réponses } \\
\text { erronées avant l'activité }\end{array}$} & \multicolumn{2}{|c|}{$\begin{array}{l}\text { Fréquence des réponses } \\
\text { correctes après l'activité }\end{array}$} & \multicolumn{2}{|c|}{$\begin{array}{c}\text { Gain conceptuel par } \\
\text { question }\end{array}$} & \multicolumn{2}{|c|}{ Tests statistiques } \\
\hline & $\mathrm{CC}$ & $\mathrm{CC}+\mathrm{EI}$ & $\mathrm{CC}$ & $\mathrm{CC}+\mathrm{EI}$ & $\mathrm{CC}$ & $\mathrm{CC}+\mathrm{EI}$ & $\chi^{2}$ & $p$ \\
\hline Q1 & $34 / 72$ & $40 / 78$ & $25 / 34$ & $35 / 40$ & $73,5 \%$ & $85,7 \%$ & 2,34 & 0,13 \\
\hline Q2 & $32 / 72$ & $38 / 78$ & $19 / 32$ & $29 / 38$ & $59,4 \%$ & $76,3 \%$ & 2,30 & 0,13 \\
\hline Q3 & $63 / 72$ & $69 / 78$ & $14 / 63$ & $35 / 69$ & $22,2 \%$ & $50,7 \%$ & 11,51 & 0,0007 \\
\hline Q4 & $41 / 72$ & $52 / 78$ & $15 / 41$ & $43 / 52$ & $36,6 \%$ & $82,7 \%$ & 20,77 & $<0,0001$ \\
\hline
\end{tabular}




\section{Discussion}

Pour améliorer les scores des apprenants de grade 10 qui répondent à des questions sur la concentration chimique, notre méthodologie a été d'utiliser le conflit perceptocognitif associé ou non à une approche métacognitive centrée sur le contrôle inhibiteur des conceptions non pertinentes.

\subsection{Les conflits cognitifs apparents réalisés}

Une petite moitié des élèves interrogés (44\%) ne prévoient pas l'augmentation du niveau de liquide après l'ajout du chlorure de sodium dans l'eau. Suite à l'activité visant un éventuel conflit cognitif, deux-tiers d'entre eux justifient correctement que le niveau monte. Deux stratégies sont mises en évidence: $47 \%$ des élèves expliquent que «les entités de sel prennent de la place donc le volume augmente » et $53 \%$ déclarent que «plus de matière entraine plus de volume ». Nous attribuons ce changement d'expression à la réutilisation de la $p$-prim «More A then more B ». Nous utilisons le terme « réutilisation » puisque lors du test initial à la remédiation, nous avons mis en évidence que la presque totalité des élèves de première primaire utilisent cette habitude spontanée et prévoient correctement l'augmentation du niveau de liquide suite à la dissolution du sel de cuisine dans l'eau.

Nous avons cherché à vérifier si un lien existe entre la stratégie acquise après l'activité de remédiation et celle que l'élève utilisait dans sa prévision, avant l'activité. Les élèves qui prévoyaient avant l'activité que le niveau ne monterait pas, car « les entités de sel s'intercalent entre les molécules d'eau » en ayant donc une vision microscopique de la matière conservent cette vision puisque 80 \% d'entre eux déclarent après l'activité que « les entités de sel prennent de la place ». D'autres élèves expriment une vision davantage macroscopique de la matière en déclarant que le niveau ne monte pas, car « le sel disparaît » ou « le sel absorbe l'eau » ou encore, selon Lavoisier, « Rien ne se perd, rien ne se gagne, tout se transforme ». Après l'activité, la plupart d'entre eux (entre 77 et $100 \%$ selon la conception de départ) admettent que le niveau monte, car «plus de matière entraine plus de volume ». Ils restent donc sur une échelle macroscopique de la matière.

Si l'on se place du point de vue du modèle de prévalence conceptuelle de Patrice Potvin (2013), un changement de prévalence semble se réaliser suite au conflit cognitif et des « liens cognitifs » privilégiés semblent exister. Une hypothèse est que les élèves qui utilisent avant l'activité une conception plus intuitive, sans référence aux modèles microscopiques de la matière (« le sel disparaît », « le sel absorbe l'eau » ou « rien ne se perd, rien ne se gagne, tout se transforme »), rendent prévalente une conception tout aussi intuitive liée directement à une p-prim ("plus de matière donc plus de volume ») et qui reste sur une vision macroscopique de la matière. Ceux qui prévoyaient que le niveau de liquide ne monte pas, en se référant à une conception impliquant davantage une visualisation microscopique de la matière
(« les entités de sel s'intercalent entre les molécules d'eau ») telle que mise en apprentissage en chimie, rendent prévalente une conception selon laquelle cette vision reste présente tout en y intégrant l'utilisation de la $p$-prim « More A then more $B$ » (« les entités de sel prennent de la place donc le volume augmente »).

\subsection{Le gain conceptuel par élève}

L'analyse des résultats montre qu'une augmentation des scores est statistiquement significative pour les élèves ayant été confrontés au conflit cognitif. La moyenne du gain conceptuel par élève pour quatre questions est de $+0,64$ dans le cas du groupe $\mathrm{CC}$; ce résultat est interprété comme le fait que deux élèves sur trois répondent correctement à une question supplémentaire sur les quatre questions après l'activité impliquant le conflit percepto-cognitif. Le conflit percepto-cognitif leur permet d'acquérir que le soluté prend de la place au sein de la solution et ainsi de concevoir que le volume de solution est supérieur au volume de solvant. Le calcul de la concentration impliquant la division par le volume total, la conception pertinente « J'utilise le volume de solution pour calculer une concentration » est donc davantage mobilisée par les apprenants.

L'ajout au conflit cognitif d'un entrainement à la fonction exécutive de contrôle inhibiteur permet d'améliorer davantage les scores. Le gain conceptuel moyen pour le groupe ayant été soumis au conflit cognitif puis à un entrainement à l'inhibition $(+1,65$ pour 4 questions) montre qu'en moyenne chaque élève répond correctement à une question supplémentaire sur quatre par rapport aux élèves du groupe conflit cognitif seul.

Cette étape supplémentaire d'automatisation à l'inhibition de la conception non pertinente permet également de réduire fortement le nombre d'élèves qui répondent moins bien au post-test: 1 élève du groupe CC+EI au lieu de 14 pour le groupe $\mathrm{CC}$ obtient un «gain» de -1 . L'objectif étant de permettre une amélioration de l'acquisition du concept de concentration pour l'ensemble des élèves, l'entrainement à l'inhibition va davantage dans ce sens.

L'acquisition du concept de concentration chimique est favorisée par un apprentissage à l'inhibition de la conception non pertinente «Vsolvant». L'activation de la conception pertinente «Vsolution » semble permettre une réactivation de la p-prim «More A then more B »dans le contexte de la dissolution d'un soluté dans un solvant.

La remédiation avec l'entrainement à l'inhibition permet de mettre l'élève au niveau métacognitif et de lui faire prendre conscience qu'il doit contrôler ses habitudes en fonction du contexte. La remédiation crée en quelque sorte, une rationalisation de la pensée intuitive. L'élève est mis en garde que des conceptions sont en compétition lorsqu'il raisonne sur un concept et qu'il doit rester maitre de la conception à utiliser de façon à la rendre prévalente dans ce 
contexte : l'apprenant est mis en conscience qu'il doit gérer les fonctions exécutives qui l'amènent à raisonner.

Cette approche est positive puisque les différentes $p$-prims et les conceptions qui en résultent ne sont pas en ellesmêmes erronées, mais c'est leur activation qui peut l'être dans certains contextes.

La répétition de cette mise en garde de l'existence d'un piège permet d'automatiser le nouveau « lien cognitif » qui aboutit à activer la conception prévalente dans le contexte de la question.

\subsection{Le gain conceptuel par question}

L'analyse des résultats montre que l'entrainement à l'inhibition permet une augmentation statistiquement significative pour les questions Q3 et Q4 des pré- et posttests. Ces deux questions demandent une mobilisation cognitive plus importante que les questions Q1 et Q2 qui se réfèrent aux définitions de la concentration chimique. Elles ne demandent pas une réflexion avancée alors que les questions Q3 et Q4 sont davantage de l'ordre du savoir-faire.

La question Q3 avait été posée dans une précédente étude diagnostique (Willame et Snauwaert, 2015) : «Une solution est préparée en dissolvant $100 \mathrm{~g}$ de chlorure de sodium $\mathrm{NaCl}$ dans $500 \mathrm{~mL}$ d'eau. Quelle est la concentration massique en $\mathrm{NaCl}$ obtenue? ». La grande majorité $(90 \%)$ des élèves interrogés répondaient de façon erronée que la concentration massique est de $200 \mathrm{~g} / \mathrm{L}$ (la réponse correcte est «Il manque des informations pour répondre » puisque seul le volume de solvant est donné). Les résultats dans cette présente étude confirment ces chiffres (132 élèves sur les 150 ne répondent pas correctement avant les activités). Nous avions suggéré que les interrogés appliquent le contrat didactique implicite en sciences «Des valeurs numériques dans un énoncé impliquent un calcul numérique dans la réponse ». Les résultats présentés dans cet article montrent que le conflit cognitif seul a permis à un élève sur quatre de répondre correctement à cette même question alors que le conflit cognitif associé au contrôle inhibiteur permet de doubler cette fréquence puisqu'un élève sur deux répond alors correctement. Le contrat didactique reste cependant appliqué par de nombreux élèves.

La question Q4 s'intéresse à la façon de préparer une solution. Le fait que le soluté prenne de la place au sein de la solution implique au chimiste d'ajouter le soluté avant de compléter son volume au volume désiré de solution. Le conflit cognitif permet à un peu plus d'un tiers des interrogés de répondre correctement, mais le contrôle inhibiteur fait évoluer ce résultat à plus de 8 élèves interrogés sur 10 . Le fait de se rendre compte, grâce à la manipulation, que le niveau de liquide augmente n'est pas suffisant pour la majorité des élèves à rendre prévalente la conception pertinente 《Vsolution ». C'est l'inhibition de la conception «Vsolvant » et l'automatisation de l'activation de la conception «Vsolution» qui permet de rendre cette conception pertinente prévalente dans le contexte de la préparation d'une solution de concentration connue.

Dans le contexte de la dissolution d'un soluté et du calcul de sa concentration au sein de la solution, nos résultats montrent l'intérêt du conflit cognitif suivi d'un entrainement à l'inhibition des conceptions non pertinentes. La fonction exécutive telle que le contrôle inhibiteur semble jouer un rôle important dans le parcours scolaire des apprenants en sciences.

Sur base du test diagnostique réalisé dans les classes de différents niveaux (de la primaire aux futurs enseignants de sciences; tableau 1), les activités proposées aux apprenants dans le cadre de cette étude permettraient une réactivation d'une habitude délaissée dans le cadre de la dissolution d'un soluté chez les plus experts en chimie : "More A then more $B$ ». Cette $p$-prim n'est pas pertinente dans tous les contextes scientifiques. Ainsi, dans le contexte de la chute des corps, cette habitude de causalité proportionnelle est celle à inhiber puisque ce n'est pas parce que la masse d'un objet est plus grande que sa vitesse de chute augmente (Brault Foisy et al., 2015 ; Dunbar et al., 2007). De même, en mathématiques, cette même habitude est également celle à inhiber dans le cas, par exemple, de la comparaison de l'aire et du périmètre de différentes figures géométriques. En effet, ce n'est pas parce qu'une surface est plus petite ou plus grande que le périmètre est proportionnellement plus petit ou plus grand (Babai et al., 2009).

La reconnaissance du contexte dans lequel le sujet doit apprendre à bloquer certaines réponses intuitives erronées est donc essentielle. Il s'agit donc d'entrainer l'apprenant dans un premier temps à reconnaître le contexte de l'apprentissage ou de la question puis, dans un second temps, à contrôler ses réponses intuitives.

\section{Conclusion et propositions pour l'enseignement}

Notre étude montre que l'apprentissage d'un concept passe aussi par un contrôle de ses intuitions, de ses habitudes intuitives pour rationaliser sa pensée. L'enseignant qui est au cœur de l'apprentissage de ses élèves a donc un rôle majeur à jouer. Il doit être celui qui participe à la prise de conscience que chacun a emmagasiné des conceptions utiles, mais qui doivent être mobilisées de façon pertinente en fonction du contexte. Il s'agit de rendre prévalente celle qui permettra d'envisager le concept de façon experte.

Pour remédier aux difficultés d'apprentissage d'un concept, le conflit cognitif qui précède l'entrainement au contrôle inhibiteur joue un rôle essentiel. L'élève en difficulté n'envisage pas la conception pertinente comme celle à rendre prévalente du fait qu'il accorde un statut trop important aux conceptions non appropriées. Le conflit lui fait prendre conscience qu'il doit modifier ce statut. Le conflit cognitif n'est pas vécu comme une arme qui attaque l'intelligence de l'individu, mais comme une lampe qui éclaire la conception à rendre prévalente. 
L'élève doit ensuite s'entrainer à allumer cette lampe tout en laissant dans l'obscurité les conceptions non adéquates dans le contexte considéré. Il devra les éclairer en fonction du contexte. Cette étape d'entrainement à l'inhibition des conceptions non pertinentes est donc fondamentale pour automatiser l'éclairage, l'activation de la conception appropriée dans le bon contexte. Les outils tels que les alertes émotives (ou «inhibitive stop signs 》) sont les signaux déclencheurs d'un contrôle de ses intuitions afin d'« attraper le piège » et mobiliser ainsi la conception pertinente. L'automatisation de ce processus permettra de changer, non pas de conception, mais de prévalence conceptuelle.

Une récente étude de Potvin et Cyr (2017), menée sur le thème de la flottabilité de boules de masse, volume et matière différentes, suggère que l'enseignement a permis de rendre prévalente les conceptions scientifiques des experts. Notre étude montre que l'enseignement peut, à l'inverse, rendre prévalentes des conceptions non appropriées. L'enseignant doit en être conscient. Il doit rester lui-même vigilant quant aux conceptions qui interfèrent et dont le statut peut être renforcé suite à la mise en apprentissage des modèles scientifiques. Cela s'avèrerait d'autant plus en chimie, où les représentations symboliques du niveau microscopique d'une matière visible macroscopiquement peuvent conduire à créer des interférences supplémentaires dont le statut trop important empêche de rendre prévalente la conception experte pour le contexte considéré.

Nous n'avons pas mesuré à long terme l'impact d'une remédiation impliquant un conflit cognitif et un apprentissage à l'inhibition de la conception non pertinente, mais il semble qu'une étape de renforcement soit nécessaire pour rendre durable la prévalence conceptuelle (Potvin et Cyr, 2017). Ce renforcement peut se faire par des retours réguliers pendant l'année sur les alertes mises en place, pendant les remédiations.

Cette étude montre que les recherches en didactique («montrer le chemin») et en sciences cognitives («comprendre le chemin») peuvent contribuer à l'amélioration de l'apprentissage en classe. La collaboration entre les chercheurs et les enseignants est essentielle pour que les outils puissent être intégrés dans les cursus scolaires.

\section{Références}

Arnaud, P. (1989). Cours de chimie physique (3 éd.). Paris, France : Dunod.

Astolfi, J.-P. (1992). Apprendre par franchissement d'obstacles? Repères, 5(1), 103-116. https://doi.org/10.3406/reper.1992.2052

Astolfi, J.-P., Darot, E., Ginsburger-Vogel, Y. et Toussaint, J. (2008). Mots-clés de la didactique des sciences ( $2^{\mathrm{e}}$ éd.). Bruxelles, Belgique : De Boeck. https://doi.org/10.3917/dbu.astol.2008.01
Babai, R., Younis, N. et Stavy, R. (2014). Involvement of inhibitory control mechanisms in overcoming intuitive interferences. Neuroeducation, 3(1), 1-9. https://doi.org/10.24046/neuroed.20140301.1

Babai, R., Zilber, H., Stavy, R. et Tirosh, D. (2009). The effect of intervention on accuracy of students' responses and reaction times to geometry problems. International Journal of Science and Mathematics Education, 8(1), 185201. https://doi.org/10.1007/s10763-009-9169-8

Borst, G., Moutier, S. et Houdé, O. (2014). Negative priming in logicomathematical reasoning: the cost of blocking your intuition. Dans W. De Neys et M. Osman (dir.), New approaches in reasoning research - Current issues in Thinking \& Reasoning (p. 34-50). New York, NY: Psychology Press.

Brault Foisy, L.-M., Potvin, P., Riopel, M. et Masson, S. (2015). Is inhibition involved in overcoming a common physics misconception in mechanics? Trends in Neuroscience and Education, 4(1-2), 26-36. https://doi.org/10.1016/j.tine.2015.03.001

Brown, D. E. (1993). Refocusing core intuitions: A concretizing role for analogy in conceptual change. Journal of Research in Science Teaching, 30(10), 1273-1290. https://doi.org/10.1002/tea.3660301009

Cassotti, M. et Moutier, S. (2010). How to explain receptivity to conjunction-fallacy inhibition training: Evidence from the Iowa Gambling Task. Brain and Cognition, 72(3), 378384. https://doi.org/10.1016/j.bandc.2009.11.004

Clement, J., Brown, D. E. et Zietsman, A. (1989). Not all preconceptions are misconceptions: finding "anchoring conceptions" for grounding instruction on students' intuitions. International Journal of Science Education, 11(5), 554-565. https://doi.org/10.1080/0950069890110507

de Berg, K. (2012). A study of first-year chemistry student' understanding of solution concentration at the tertiary level. Chemistry Education Research and Practice, 13(1), 816. https://doi.org/10.1039/c1rp90056k

Damasio, A. R. (1994). Descartes' Error: Emotion, reason, and the human brain. New York, NY : Grosset, Putman.

Devetak, I., Vogrinc, J. et Glažar, S. A. (2008). Assessing 16year-old students' understanding of aqueous solution at submicroscopic level. Research in Science Education, 39(2), 157-179. https://doi.org/10.1007/s11165-007-9077-2

diSessa, A. A. (1983). Phenomenology and evolution of intuition. Dans D. Gentner et A. L. Stevens (dir.), Mental models (p. 15-33). Mahwah, NJ : Lawrence Erlbaum.

diSessa, A. A. (2006). A history of conceptual change research: Threads and fault lines. Dans R. K. Sawyer (dir.), The Cambridge handbook of the learning sciences (p. 265-281). Cambridge, Royaume-Uni : Cambridge University Press. 
diSessa, A. A. (2013). A bird's-eye view of the "Pieces" vs. "Coherence" controversy (From the "Pieces" side of the fence). Dans S. Vosniadou (dir.), International handbook of research on conceptual change ( $2^{\mathrm{e}}$ éd., p. 31-48). New York, NY: Routledge.

https://doi.org/10.4324/9780203154472.ch2

Duit, R. et Treagust, D. F. (2003). Conceptual change: A powerful framework for improving science teaching and learning. International Journal of Sciences Education, 25(6), 671-688. https://doi.org/10.1080/09500690305016

Dunbar, K. N., Fugelsang, J. A. et Stein, C. (2007). Do naive theories ever go away? Using brain and behavior to understand changes in concepts. Dans M. C. Lovett et P. Shah (dir.), Thinking with Data: 33'd Carnegie Symposium on Cognition (p. 193-206). Mahwah, NJ : Erlbaum.

Fugelsang, J. A. et Dunbar, K. N., (2005). Brain-based mechanism underlying complex causal thinking. Neuropsychologia, 43(8), 1204-1213.

https://doi.org/10.1016/j.neuropsychologia.2004.10.012

Gandillet, E. et Le Maréchal, J.-F. (2003, octobre). Conceptions et chimie des solutions ioniques. Actes de colloque des $3^{\text {es }}$ Rencontres scientifiques de l'ARDiST, Toulouse.

Khang, G. N. et Sai, C. L. (1987). Secondary school students' difficulties in learning the "Mole Concept" - A preliminary study in Singapore. Singapore Journal of Education, 8(1), 80-88. https://doi.org/10.1080/02188798708547617

Houdé, O. (2016a). Apprendre à résister aux automatismes. Cahiers pédagogiques, (527), 20-22.

Houdé, O. (2016b, mai). Comment aider les élèves à inhiber leurs erreurs scolaires persistantes. Conférence présentée au $5^{\mathrm{e}}$ Congrès de l'Association pour la recherche en neuroéducation, UQAM, Montréal (Canada), 24-27 mai 2016.

Houdé, O. (2004). La psychologie de l'enfant ( 6 éd.). Paris, France : Presses Universitaires de France.

Houdé, O. et Tzourio-Mazoyer, N. (2003). Neural foundations of logical and mathematical cognition. Nature Reviews Neuroscience, 4(6), 507-514. https://doi.org/10.1038/nrn1117

Houdé, O., Zago, L., Crivello, F., Moutier, S., Pineau, A., Mazoyer, B. et Tzourio-Mazoyer, N. (2001). Access to deductive logic depends on a right ventromedial prefrontal area devoted to emotion and feeling: Evidence from a training paradigm. NeuroImage, 14(6), 1486-1492. https://doi.org/10.1006/nimg.2001.0930

Houdé, O., Zago, L., Mellet, E., Moutier, S., Pineau, A., Mazoyer, B. et Tzourio-Mazoyer, N. (2000). Shifting from the perceptual brain to the logical brain: The neural impact of cognitive inhibition training. Journal of Cognitive Neurosciences, 12(5), 721-728.

https://doi.org/10.1162/089892900562525
International Union of Pure and Applied Chemistry (IUPAC). (2016). La dissolution d'un cristal de sel dans l'eau. http://old.iupac.org/didac/Didac\%20Fr/Didac01/Con tent/H04\%20-\%20H05.htm

Johnstone, A. H. (1993). The development of chemistry teaching: A changing response to changing demand. Journal of Chemical Education, 70(9), 701-705. https://doi.org/10.1021/ed070p701

Kuhn, T. S. (1983). La structure des révolutions scientifiques. Paris, France : Flammarion.

Legrand, J.-C. (2006). Cours de thermodynamique. Résumé. Formation d'ingénieurs en Matériaux. Ecole polytechnique universitaire Pierre et Marie Curie. http://www.edu.upmc.fr/chimie/jclegrand/partie1_0 6_07.pdf

Lubin, A., Lanoë, C., Pineau, A. et Rossi, S. (2012). Apprendre à inhiber: Une pédagogie innovante au service des apprentissages scolaires fondamentaux (mathématiques et orthographe) chez des élèves de 6 à 11 ans. Neuroeducation, 1(1), 55-84. https://doi.org/10.24046/neuroed.20120101.55

Masson, S. (2012). Etude des mécanismes cérébraux liés à l'expertise scientifique en électricité à l'aide de l'imagerie par résonance magnétique fonctionnelle. Thèse de doctorat inédite. Université du Québec à Montréal, Canada. http://www.archipel.uqam.ca/4876/1/D2287.pdf

Masson, S. (2016, mai). Cerveau, apprentissage et enseignement des mathématiques et des sciences: Comment aider les élèves à surmonter leurs difficultés? Conférence présentée au $5^{\mathrm{e}}$ Congrès de l'Association pour la recherche en neuroéducation, UQAM, Montréal (Canada), 24-27 mai 2016.

Minstrell, J. (2001). Facets of students' thinking: Designing to cross the gap from research to standards-based practice. Dans K. Crowley, C. D. Schunn et T. Okada (dir.), Designing for science: Implications for professional, instructional, and everyday science. Mahwah, $\mathrm{NJ}$ : Lawrence Erlbaum Associates.

Nivaldo, J. T. (2011). Chemistry: A molecular approach ( $2^{\mathrm{e}}$ éd.). Upper Saddle River, NJ : Pearson Prentice Hall.

Nussbaum, J. et Novick, S. (1982). Alternative frameworks, conceptual conflict and accommodation: Toward a principled teaching strategy. Instructional Science, 11(3), 183-200. https://doi.org/10.1007/bf00414279

Orange, C. et Orange-Ravachol, D. (2013). Le concept de représentation en didactique des sciences : Sa nécessaire composante épistémologique et ses conséquences. Recherches en éducation, 17, 46-61.

Posner, G. J., Strike, K. A., Hewson, P. W. et Gertzog, W. A. (1982). Accomodation of a scientific conception: Toward a theory of conceptual change. Science Education, 66(2), 211-227. https://doi.org/10.1002/sce.3730660207 
Potvin, P. (2013). Proposition for improving the classical models of conceptual change based on neuroeducational evidence: Conceptual prevalence. Neuroeducation, 2(1), 16-43. https://doi.org/10.24046/neuroed.20130201.16

Potvin, P. (2011). Manuel d'enseignement des sciences et de la technologie: Pour intéresser les élèves $d u$ secondaire. Québec, Canada : MultiMondes.

http://multim.com/titre/?ID=350

Potvin, P. et Cyr, G. (2017). Toward a durable prevalence of scientific conceptions: Tracking the effects of two interfering misconceptions about buoyancy from preschoolers to science teachers. Journal of Research in Science Teaching, 54(9), 1121-1142.

https://doi.org/10.1002/tea.21396

Potvin, P., Riopel, M. et Masson, S. (2007). Regards multiples sur l'enseignement des sciences. Québec, Canada: MultiMondes. http://multim.com/titre/?ID=212

Potvin, P., Sauriol, E. et Riopel, M. (2015). Experimental evidence of the superiority of the prevalence model of conceptual change over classical models and repetition. Journal of Research in Science Teaching, 52(8), 1082-1108. https://doi.org/10.1002/tea.21235

Reuter, Y., Cohen-Azria, C., Daunay, B., Delcambre, I. et Lahanier-Reuter, D. (2013). Dictionnaire des concepts fondamentaux des didactiques ( $3^{\mathrm{e}}$ éd.). Bruxelles, Belgique : De Boeck.

https://doi.org/10.3917/dbu.reute.2013.01

Rossi, S., Lubin, A., Lanoë, C. et Pineau, A. (2012). Une pédagogie du contrôle cognitif pour l'amélioration de l'attention à la consigne chez l'enfant de 4-5 ans. Neuroeducation, 1(1), 29-54. https://doi.org/10.24046/neuroed.20120101.29

Siegler, R. S. (1996). Emerging minds. The process of change in children's thinking. New York, NY: Oxford University Press.

Smith, C. A. et Lazarus, R. S. (1990). Emotion and adaptation. Dans L. A. Pervin (dir.), Handbook of Personality: Theory and Research (p. 609-637). New York, NY : Guilford.

Stavy R. et Tirosh D. (2000). How students (mis-)understand science and mathematics. Intuitive rules. New York, NY : Teachers College Press.

Strike, K. A. et Posner, G. J. (1992). A revisionist theory of conceptual change. Dans R. A. Duschl et R. J. Hamilton (dir.), Philosophy of science, cognitive psychology, and educational theory and practice (p. 147-176). Albany, NY : SUNY Press.

Tsaparlis, G. et Sevian, H. (dir.). (2013). Concepts of matter in science education. Dordrecht, Pays-Bas : Springer. https://doi.org/10.1007/978-94-007-5914-5
Tsoumpelis, L. et Gréa, J. (1995). Essai d'application de la théorie des situations en sciences physiques Apprentissage de la concentration molaire en classe de première S. Recherches en Didactique des Mathématiques, 15(2), 63-108. http://rdm.penseesauvage.com/Essai-dapplication-de-la-theorie.html

Tümay, H. (2016). Reconsidering learning difficulties and misconceptions in chemistry: Emergence in chemistry and its implications for chemical education. Chemistry Education Research and Practice, 17(2), 229-245. https://doi.org/10.1039/c6rp00008h

Van Lerberghe, L. (2017). Des conceptions naïves aux conceptions expertes grâce à un apprentissage alliant contrôle inhibiteur et conflit cognitif. Mise en application au travers de l'acquisition du concept de concentration massique en troisième générale option sciences. Mémoire de master en sciences de l'éducation. Université catholique de Louvain, Louvain-la-Neuve, Belgique. https://dial.uclouvain.be/memoire/ucl/fr/object/the sis\%3A8550

Vermersch, P. (2011). L'entretien d'explicitation ( $7^{e}$ éd.). Issyles-Moulineaux : ESF.

Viennot, L. (2008). Learning and conceptual understanding: Beyond simplistic ideas, what have we learned? International Commission on Physics Education. http://web.phys.ksu.edu/ICPE/Publications/teach2/ Viennot.pdf

Vosniadou, S. et Brewer, W. F. (1992). Mental models of the earth: A study of conceptual change in childhood. Cognitive Psychology, 24(4), 535-585. https://doi.org/10.1016/0010-0285(92)90018-w

Willame, B. (2017). Construction d'outils didactiques pour remédier aux difficultés d'apprentissage du concept de concentration en chimie dans le secondaire supérieur. Appui sur les neurosciences cognitives. Thèse de doctorat en didactique des sciences. Université de Namur. Namur, Belgique : Presses Universitaires de Namur. https://pure.unamur.be/ws/portalfiles/portal/35494 $\underline{384}$

Willame, B. et Snauwaert, P. (2015). Les difficultés rencontrées dans l'apprentissage du concept de concentration en chimie. Spiral-E. Revue de recherches en éducation, 55(1), 177-205. https://doi.org/10.3406/spira.2015.1743

Willame, B. et Snauwaert, P. (2016). Et si nous mettions du sel dans l'eau - Comment évoluent les conceptions des apprenants au fur et à mesure des apprentissages en chimie? Spectre, Revue de l'Association pour l'enseignement de la science et de la technologie au Québec, 45(3), 18-21. 


\section{ANNEXE 1}

\section{Pré-test}

1. Quelle expression correspond le mieux au concept de « concentration chimique » :

a) Quantité de soluté dans un volume de solvant

b) Rapport entre une quantité de soluté et le volume de solution*

c) Grande quantité de soluté dans une solution

d) Molécules de soluté qui se regroupent dans un solvant

e) Aucune des possibilités

f) Je ne sais pas

2. Dans l'expression de la concentration $\mathrm{C}$, que représente $\mathrm{V}$ ?
a) Le volume de solution*
b) Le volume de solvant
c) Le volume de soluté
d) Aucune des possibilités
e) Je ne sais pas

3. Une solution est préparée en dissolvant $100 \mathrm{~g}$ de chlorure de sodium $\mathrm{NaCl}$ dans $500 \mathrm{~mL}(0,500 \mathrm{~L})$ d'eau. Quelle est la concentration massique en $\mathrm{NaCl}$ obtenue ?
a) $0,200 \mathrm{~g} / \mathrm{L}$
b) $200 \mathrm{~g} / \mathrm{L}$
c) $5,00 \mathrm{~mL} / \mathrm{g}$
d) Il manque des informations pour répondre*
e) Aucune des possibilités

4. Pour préparer une solution aqueuse de chlorure de sodium ( $\mathrm{NaCl}$ dans l'eau) à $2 \mathrm{~g} / \mathrm{L}$, que faut-il faire ?
a) Placer $1 \mathrm{~L}$ d'eau dans une fiole et ajouter $2 \mathrm{~g}$ de $\mathrm{NaCl}$
b) Placer $2 \mathrm{~g}$ de $\mathrm{NaCl}$ dans une fiole et ajouter $1 \mathrm{~L}$ d'eau
c) Placer $2 \mathrm{~g}$ de $\mathrm{NaCl}$ dans une fiole et compléter avec de l'eau jusqu'à obtenir un volume de $1 \mathrm{~L}^{*}$
d) Les propositions a ou b parce que l'ordre d'ajout du soluté n'a pas d'importance
e) Aucune des possibilités
f) Je ne sais pas 


\section{ANNEXE 2}

\section{Outil visant le conflit cognitif}

Lis attentivement toutes les indications données, sans passer aucune ligne.

Le matériel nécessaire : Un porte tube avec

- 1 tube à essai contenant de l'eau dont le niveau est indiqué par un trait

- 1 tube à essai contenant du sel de cuisine

Complète les encadrés au fur et à mesure que cela est demandé sans revenir en arrière une fois l'étape complétée.

\section{Étape 1. Prévisions}

1.1. Si la totalité du sel contenu dans le tube à essai était versée dans l'eau et que l'agitation permettait la dissolution complète du sel, que ferait le niveau du liquide?

Choisis l'une des propositions :

Le niveau reste le même

Le niveau diminue en dessous du trait

Le niveau monte au dessus du trait

1.2. Explique ton choix en étant le plus explicite possible :

\section{Étape 2. Observations}

2.1. Verse maintenant la totalité du sel dans l'eau, bouche le tube avec le bouchon puis mélange pendant 30 secondes en bloquant le bouchon avec un doigt.

2.2. Que fait le niveau de liquide? Choisis l'une des propositions :

Le niveau reste le même

Le niveau diminue en dessous du trait

Le niveau monte au dessus du trait

2.3. Compare ce résultat à ta prévision. Avais-tu raison? oui ou non

Si tu as répondu «non » : comment expliques-tu l'évolution réelle du niveau de liquide? 
